\title{
Design and Expansion Planning of Parallel Inverter Based AC Microgrids - An Approach for Improved Stability Margins
}

\author{
Ayesha Firdaus*, Student Member, IEEE, Sukumar Mishra*, Senior Member, IEEE, Chen Zhang ${ }^{\dagger}$, and Marta \\ Molinas $^{\dagger}$, Member, IEEE
}

\begin{abstract}
This paper presents an application of impedancebased modelling approach to identify suitable locations for design and expansion planning of an inverter based autonomous AC microgrid. The objective is to find out ideal location for placement of additional components which can include new inverter-based sources, microgrid damping controller, etc., ensuring sufficient stability margins. These objectives are achieved by first identifying the weakest and the strongest nodes in the system with respect to the system stability and then observing the effects of choosing various nodes for design and expansion on overall system stability. The weakest and the strongest nodes are identified using the impedance based stability analysis of the microgrid by dividing the system in various possible partition points while the impact of choosing different locations on stability is analyzed by eigen value analysis as well as time-domain MATLAB/Simulink simulations and experimental results.
\end{abstract}

Index Terms-Distributed generation, dynamic stability, impedance-based stability analysis, microgrid, suitable location, stability margins.

\section{NOMENCLATURE}

$\delta$

$\omega \quad$ Operating frequency of DG

$\omega_{c} \quad$ Cut-off frequency of low pass filter

$\omega_{n} \quad$ Nominal frequency set point of DG

$\theta \quad$ Reference phase

$F \quad$ Feed-forward gain of voltage controller

$m, n \quad$ Active and reactive power droop coefficients

$V_{n} \quad$ Nominal d-axis voltage set point of DG

$i_{l d}^{*}, i_{l q}^{*} \quad \mathrm{dq}$ components of reference coupling inductor current of inverter

$i_{l d}, i_{l q} \quad \mathrm{dq}$ components of inductor coupling current

$i_{\text {line } D Q} \mathrm{dq}$ component of connecting line currents

$i_{\text {loadDQ }} \quad \mathrm{dq}$ component of load currents

$i_{o d}, i_{o q}$ dq components of output current of inverter

$K_{p c}, K_{i c} \quad$ Propotional and integral gain of current controller of grid forming inverter

$K_{p p}, K_{i p} \quad$ Propotional and integral gain of power controller of grid feeding inverter

$K_{p v}, K_{i v} \quad$ Propotional and integral gain of voltage controller of grid forming inverter

* Ayesha Firdaus, and Sukumar Mishra are with the Department of Electrical Engineering, Indian Institute of Technology Delhi, India.

†Chen Zhang, and Marta Molinas are with the Department of Engineering Cybernetics, NTNU, 7034, Trondheim, Norway.
$K_{p}^{P L L}, K_{i}^{P L L}$ Propotional and integral gain PLL

$K_{p}, K_{i} \quad$ Propotional and integral gain of current controller of grid feeding inverter

$L_{c}, r_{c} \quad$ Inductance and resistance of coupling branch of inverter

$L_{f}, r_{f}, C_{f} \quad$ Per phase inductance, resistance and capacitance of inverter filter

$L_{\text {Line }}, R_{\text {line }}$ Inductance and resistance of connecting lines

$L_{\text {Load }}, R_{\text {Load }}$ Inductance and resistance of loads

$P, Q \quad$ Average value of active and reactive power

$p, q \quad$ Instantaneous value of active and reactive power

$v_{c d}^{*}, v_{c q}^{*} \quad \mathrm{dq}$ components of reference output voltage of inverter

$v_{c d}, v_{c q} \quad \mathrm{dq}$ components of output voltage of inverter

$v_{i d}^{*}, v_{i q}^{*} \quad \mathrm{dq}$ components of reference voltage of inverter

$v_{i d}, v_{i q} \quad \mathrm{dq}$ components of inverter voltage

\section{INTRODUCTION}

$\mathbf{W}$ ITH the increase in the renewable energy penetration to enhance the reliability of the system, power electronic technology based voltage source inverters (VSIs) are emerging as an important interface between renewable energy based distribured generation (DG) and loads within a microgrid. These microgrid systems have the capability to operate with conventional grids (grid connected mode) as well as in isolation (autonomous mode). When microgrids are working in isolation, grid forming inverters which work on droop philosophy are usually used as interfacing devices to supply local loads [1]. Since these inverters generate their own frequency and control the bus voltages, they have different control structure from the ones that work in grid connected mode (grid feeding inverter) [2].

When the grid forming VSI based DGs are operated in autonomous mode, system stability needs attention due to lesser inertia of DGs which will lead to control mode oscillations [1], [2]. State space modelling based small signal stability analysis has been extensively used to define the stability margins of AC microgrids. Effects of controller gains, filter parameters, connecting line parameters, load parameters, etc. have been very well reported in literature [3]-[5]. Previous studies show that microgrids suffer from poorly damped low frequency oscillations mainly affected by droop controller and network dynamics [6]. However, voltage and current controller as well as filter parameters are responsible for medium and 
high frequency oscillations which have significant damping. Different type of loads also affect the microgrid stability margins differently, e.g. large induction motor type dynamic load degrades the low frequency stability of microgrids [7]. However, small induction motors affects the medium frequency oscillations in microgrid [8]. Constant power loads (CPL) doesn't affect the low frequency modes but they interact with the medium frequency modes to make the system stability critical [9]-[11]. Due to the sensitivity of oscillations with system parameters, many authors have come up with various optimization algorithms for optimal gain tuning of inverter controls to get the maximum margins [12], [13]. Various control techniques have also been applied to damp out the critical modes in the microgrid for small signal stability enhancement [14]-[19]. From the extensive studies so far, it is found that autonomous microgrids suffer from lower stability margins which requires proper design and control of the system.

To perform the stability analysis of inverter fed systems in a computationally effective manner, impedance-based stability analysis is also extensively used [20]-[25]. Impedance is an effective way to represent power electronic converter based system [26]. It can be found via linearisation or by measurements. Power electronic converters and other power system components can be represented as equivalent small signal impedance which is viewed as terminal equivalent model [27]. Since, other stakeholders and operators are concerned to the terminal behaviour of components only and they don't require any internal configuration of the components, impedance based analysis is useful over state space method [28]. Impedance based stability analysis using generalized Nyquist criterion (GNC) has been extensively used for grid feeding inverters and CPLs [29]-[32]. It is found that constant power converters (similar to tightly controlled inverters) provide limited stability margins in low and mid frequency region due to their negative incremental resistance when they act as source or load. Application of impedance based analysis for droop controlled inverters is also investigated in [33]-[36], where emphasis is to develop an impedance model which can capture lower frequency oscillations. It has been found that impedance based analysis is highly sensitive to partition points and it has natural interactions with circuit laws which makes it a powerful tool to analyse the network easily [37]. This characteristics of impedance influenced the authors to use it for stability oriented network design of future microgrids.

\section{A. Discussion}

Numerous modelling approaches and analysis have been presented for defining the stability margins of droop controlled inverters. Accepting the low inertia of distributed generation, IEEE Std. 1547 requires stability assessment to define the margins by the system operators. For expansion of installed microgrid system, design and planning engineer also require prior knowledge of stability margins at different part of the system. This enables the operator to know which bus in a system is more suitable to have a new source to be connected in terms of stability so that expansion of microgrids can be well planned. So far, all the studies presented are mainly focussed on analysing the behaviour of various components on system stability, improving the transient load sharing or damping of low frequency oscillations to provide larger stability margins to microgrid under different operating scenarios. Effect of component location in a network on system stability has not been explored much. This problem in droop controlled microgrids is not reported in literature and hence makes the basis of the this paper. This work establish a method for identifying the system's weakest/strongest stable location so that a better network expansion planning can be finally achieved. The paper focusses on the design and expansion procedure so as to get maximum stability margins for microgrid. This paper is divided into two parts. First is the identification of critical points in a network in terms of stability margins and the second part explains the usefulness of these points in view of network design and planning.

\section{B. Contribution}

The key contributions of this work are listed as follows:

- To find the weakest and strongest node in an autonomous system and also to identify possible sources of current instability problems that are observed in the network.

- To find ideal location for placement of damping controller with reference to these weakest and strongest nodes.

- To find ideal location for placement of grid forming inverter with reference to these weakest and strongest nodes.

- To find ideal location for placement of grid following inverter with reference to these weakest and strongest nodes.

\section{Organization}

The remainder of this paper is organized as follows. Section II gives an insight to the test microgrid under study. Section III presents the impedance modelling of grid forming inverters. Identification and analysis of weakest and strongest points in a network is presented in Section IV. Stability oriented design and planning of microgrids along with simulation results are presented in Section V. Experimental validation is presented in Section VI. Finally, the paper is concluded in Section VII.

\section{TEST MICROGRID}

Fig. 1a shows the $220 \mathrm{~V}$ (per phase RMS) test microgrid under study. There are three grid forming inverters (droop controlled) working under parallel operation to supply local loads. The inverters are responsible to maintain frequency and to control voltage at each bus. Cascaded droop, voltage and current control is employed to share active and reactive power while maintaining voltage and frequency [2]. The complete block diagram of inverter control is shown in Fig. 1b. The inverters are terminated by LC filter and are coupled to the bus through a coupling branch. There are two distribution feeders making a radial AC microgrid. The system and inverter parameters are given in Appendix. 


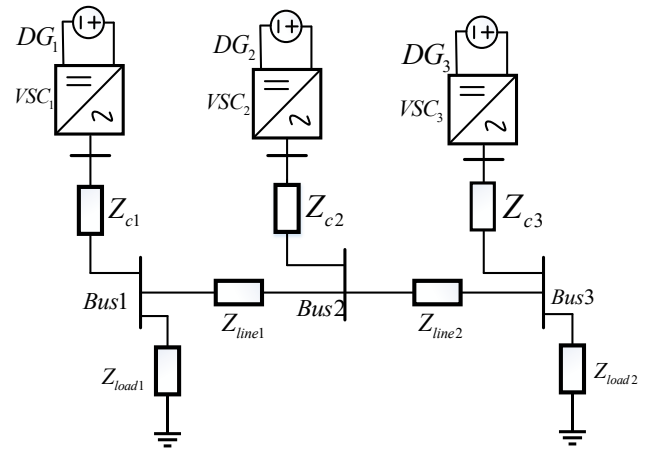

(a) Test microgrid

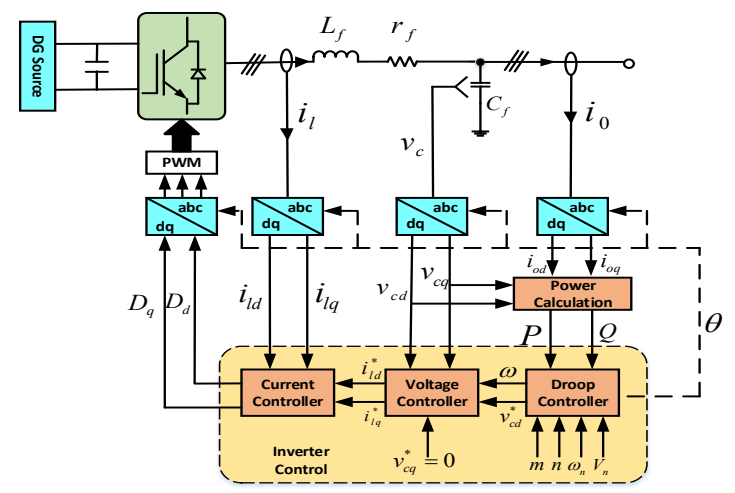

(b) Control of a grid forming inverter

Fig. 1: Autonomous microgrid system configuration and its inverter controller

\section{IMPEdAnCE-BASEd MOdELLING OF GRID FoRMING VSI}

Impedance based model of grid forming inverter is obtained using small-signal linearisation around an operating point in $d-q$ based on the model given in [8]. The output instantaneous active and reactive power supplied by the inverter to the AC bus is given by:

$$
\begin{aligned}
& p=1.5\left(v_{c d} i_{o d}+v_{c q} i_{o q}\right) \\
& q=1.5\left(v_{c d} i_{o q}-v_{c q} i_{o d}\right)
\end{aligned}
$$

A low pass filter (LPF) having cut-off frequency of $\omega_{c}$ is used to get fundamental component corresponding to inverters power. Therefore,

$$
\begin{aligned}
& P=\frac{\omega_{c}}{s+\omega_{c}} p=G_{L P}(s) p \\
& Q=\frac{\omega_{c}}{s+\omega_{c}} q=G_{L P}(s) q
\end{aligned}
$$

To mimic the governor in conventional generators, artificial droop characteristics are employed to share active and reactive powers between inverters. Droop equations are given as follows:

$$
\begin{gathered}
\omega=\omega_{n}-m P \\
v_{c d}^{*}=V_{n}-n Q \quad ; \quad v_{c q}^{*}=0
\end{gathered}
$$

Usually the inverter output voltage is aligned to $d$ axis only and hence $v_{c q}^{*}=0$.
The voltage and current controller dynamics with the inverter operating frequency $\omega$ are defined as:

$$
\begin{gathered}
i_{l d}^{*}=F i_{o d}-\omega C_{f} v_{c q}+G_{v}(s)\left(v_{c d}^{*}-v_{c d}\right) \\
i_{l q}^{*}=F i_{o q}+\omega C_{f} v_{c d}+G_{v}(s)\left(v_{c q}^{*}-v_{c q}\right) \\
v_{i d}^{*}=-\omega L_{f} i_{l q}+G_{i}(s)\left(i_{l d}^{*}-i_{l d}\right) \\
v_{i q}^{*}=\omega L_{f} i_{l d}+G_{i}(s)\left(i_{l q}^{*}-i_{l q}\right)
\end{gathered}
$$

Neglecting switching dynamics, inverter output voltage $\left(v_{i d q}\right)$ is assumed to be equal to its reference value $\left(v_{i d q}^{*}\right)$. Transfer function of voltage and current PI controllers are defined respectively as:

$$
G_{v}(s)=K_{p v}+K_{i v} / s \quad ; \quad G_{i}(s)=K_{p i}+K_{i i} / s
$$

The current and voltage dynamics of LC filter at the inverter terminals are defined as:

$$
\begin{gathered}
v_{i d}-v_{c d}=\left(r_{f}+s L_{f}\right) i_{l d}-\omega L_{f} i_{l q} \\
v_{i q}-v_{c q}=\left(r_{f}+s L_{f}\right) i_{l q}+\omega L_{f} i_{l d} \\
i_{l d}-i_{o d}=s C_{f} v_{c d}-\omega C_{f} v_{c q} \\
i_{l q}-i_{o q}=s C_{f} v_{c q}+\omega C_{f} v_{c d}
\end{gathered}
$$

The $d$ and $q$ axis output voltage equation of filter (12) \& (13) and current controller (9) \& (10) are solved together to remove the coupling terms and the resulting $d$ and $q$ axis current output is written in matrix notation as:

$$
\left[\Delta i_{l d}^{*} \Delta i_{l q}^{*}\right]^{T}=[K 1]_{2 \times 2}\left[\Delta i_{l d} \Delta i_{l q}\right]^{T}+[K 2]_{2 \times 2}\left[\Delta \omega \Delta v_{c d}^{*}\right]^{T}
$$

Now by applying small perturbation on (7) \& (8), we get:

$$
\begin{aligned}
{\left[\Delta i_{l d}^{*} \Delta i_{l q}^{*}\right]^{T}=} & {[K 3]_{2 \times 2}\left[\Delta v_{c d} \Delta v_{c q}\right]^{T}+[K 4]_{2 \times 2}\left[\Delta i_{o d} \Delta i_{o q}\right]^{T} } \\
& +[K 5]_{2 \times 2}\left[\Delta \omega \Delta v_{c d}^{*}\right]^{T}
\end{aligned}
$$

Similarly (14) \& (15) will give:

$$
\begin{aligned}
{\left[\Delta i_{l d} \Delta i_{l q}\right]^{T}=} & {[K 6]_{2 \times 2}\left[\Delta i_{o d} \Delta i_{o q}\right]^{T}+[K 7]_{2 \times 2}\left[\Delta v_{c d} \Delta v_{c q}\right]^{T} } \\
& +[K 8]_{2 \times 2}\left[\Delta \omega \Delta v_{c d}^{*}\right]^{T}
\end{aligned}
$$

The same procedure is done for droop controller dynamics (1)-(6) which will lead to:

$$
\left[\Delta \omega \Delta v_{c d}^{*}\right]^{T}=[K 9]_{2 \times 2}\left[\Delta v_{c d} \Delta v_{c q}\right]^{T}+[K 10]_{2 \times 2}\left[\Delta i_{o d} \Delta i_{o q}\right]^{T}
$$

Putting $\left[\Delta i_{l d} \Delta i_{l q}\right]^{T}$ from (18) to (16) will result in an equation in which value of $\left[\Delta i_{l d}^{*} \Delta i_{l q}^{*}\right]^{T}$ is substituted from (17). The resulting equation is given as:

$$
\begin{aligned}
& ([K 5]-[K 2]-[K 1][K 8])\left[\Delta \omega \Delta v_{c d}^{*}\right]^{T} \\
& +([K 3]-[K 1][K 7])\left[\Delta v_{c d} \Delta v_{c q}\right]^{T} \\
& =([K 1][K 6]-[K 4])\left[\Delta i_{o d} \Delta i_{o q}\right]^{T}
\end{aligned}
$$

Substitution of the dynamics of droop controller from (19) to (20) will lead to an equation relating output $\mathrm{AC}$ voltage to injected AC current and is given as:

$$
\begin{aligned}
{\left[\Delta v_{c d} \Delta v_{c q}\right]^{T} } & =[J i]_{2 \times 2}^{-1}[K i]_{2 \times 2}\left[\Delta i_{o d} \Delta i_{o q}\right]^{T} \\
& =\left[Z_{I N V}\right]_{T(2 \times 2)}\left[\Delta i_{o d} \Delta i_{o q}\right]^{T}
\end{aligned}
$$

where, $\left[Z_{I N V}\right]_{T(2 \times 2)}$ is called the $d q$ domain output impedance of grid forming inverter in an isolated AC microgrid. All the coefficient matrices are defined in Appendix. 


$$
\left[Z_{I N V}\right]_{T}=\left[\begin{array}{ll}
Z_{d d}(s) & Z_{d q}(s) \\
Z_{q d}(s) & Z_{q q}(s)
\end{array}\right]
$$

Since all the inverters are identical in the microgrid shown in Fig. 1a, the output impedance remains same for all three of them. For simplicity and for better partition point scanning, an approximated source impedance is used further which assumes zero active and reactive power droop coefficients $(m=n=$ 0 ) [30]. This assumption leads to zero value of coefficient matrices [K9] and [K10]. This assumption is valid in this work since, the main idea of representing inverters as impedance is to analyse the network through circuit laws and it depends mainly on the configuration of network. Moreover, there is not much difference in actual and approximated source impedance as also stated in [8].

\section{IDENTIFICATION OF WEAKEST AND STRONGEST POINT IN A NETWORK}

The basic idea in finding weakest and strongest point in a network is to analyse the complete network by impedance so that basic circuit laws can be applied for its reduction. The coupling branches, interconnecting line and loads are also represented in impedance form which are given as follows:

$$
\begin{aligned}
& s i_{\text {odq } i}=\left(-R_{c} / L_{c}\right) i_{\text {odq } i} \pm \omega i_{\text {odq } i}+\left(1 / L_{c}\right)\left(v_{c d q i}-v_{b d q i}\right) \\
& s i_{\text {Line } D Q i}=\left(-R_{\text {Line }} / L_{\text {Line }}\right) i_{\text {LineDQ }} \pm \omega i_{\text {Line } Q i} \\
&+\left(1 / L_{\text {Line }}\right)\left(v_{b D Q j}-v_{b D Q k}\right) \\
& s i_{\text {LoadDQ }}=\left(-R_{\text {Load }} / L_{\text {Load }}\right) i_{\text {LoadDQ } i} \pm \omega i_{\text {LoadDQ }} \\
&+\left(1 / L_{\text {Load }}\right) v_{b D Q i}
\end{aligned}
$$

where, $i$ denotes the bus number and $v_{b d q i}$ is the bus voltage at each bus. After linearisation of (23)-(25) around an operating point and representing resultant equation in the impedance form will lead to the impedance of coupling branch, interconnecting line and loads as follows:

$$
\begin{gathered}
{\left[Z_{c}\right]=\left[\begin{array}{cc}
r_{c}+s L_{c} & -\omega L_{c} \\
\omega L_{c} & r_{c}+s L_{c}
\end{array}\right]} \\
{\left[Z_{\text {Line }}\right]=\left[\begin{array}{cc}
R_{\text {Line }}+s L_{\text {Line }} & -\omega L_{\text {Line }} \\
\omega L_{\text {Line }} & R_{\text {Line }}+s L_{\text {Line }}
\end{array}\right]} \\
{\left[Z_{\text {Load }}\right]=\left[\begin{array}{cc}
R_{\text {Load }}+s L_{\text {Load }} & -\omega L_{\text {Load }} \\
\omega L_{\text {Load }} & R_{\text {Load }}+s L_{\text {Load }}
\end{array}\right]}
\end{gathered}
$$

Now, the impedance of grid forming VSI at each bus can be calculated as a series combination of source impedance at its terminal derived in previous section $\left(\left[Z_{I N V}\right]_{T}\right)$ and impedance of coupling branch.

$$
\left[Z_{I N V}\right]=\left[Z_{I N V}\right]_{T}+\left[Z_{c}\right]
$$

After representing each element by its equivalent impedance, GNC criterion is used for stability analysis [37]. For the same, source and load equivalents at each partition bus are calculated using circuit analysis as shown in Fig. 2. Suppose bus 1 is considered as partition node, then inverter at that bus is taken as source and all other impedances are reduced to get the load equivalent as shown in Fig. 2a.
TABLE I: Comparison of relative strengths of partition points

\begin{tabular}{|c|c|c|}
\hline Partition points & Phase Margin (Degrees) & Gain Margin (dB) \\
\hline Bus-1 & 45.15 & 6.86 \\
\hline Bus-2 & 33.62 & 5.946 \\
\hline Bus-3 & Inf & 23.14 \\
\hline
\end{tabular}

Similarly, one by one all buses are considered as partition points and source and load equivalents are calculated using (30) $-(33)$.

$$
\left[Z_{\text {Source }}\right]_{d q}=\left[Z_{I N V i}\right] \text { at bus } i
$$

at bus 1 :

$$
\begin{aligned}
{\left[Y_{\text {Load }}\right]_{d q} } & =\left[\left(\left(\left(\left(\left(Z_{\text {INV3 }} \| Z_{\text {Load } 2}\right)\right.\right.\right.\right.\right. \\
& \left.\left.\left.\left.\left.+Z_{\text {Line } 2}\right) \| Z_{\text {INV } 2}\right)+Z_{\text {Line } 1}\right) \| Z_{\text {Load } 1}\right)\right]^{-1}
\end{aligned}
$$

at bus 2:

$$
\begin{aligned}
{\left[Y_{\text {Load }}\right]_{d q} } & =\left[\left(\left(Z_{I N V 3} \| Z_{\text {Load } 2}\right)\right.\right. \\
& \left.\left.+Z_{\text {Line } 2}\right) \|\left(\left(Z_{I N V 1} \| Z_{\text {Load } 1}\right)+Z_{\text {Line } 1}\right)\right]^{-1}
\end{aligned}
$$

at bus 3:

$$
\begin{aligned}
{\left[Y_{\text {Load }}\right]_{d q} } & =\left[\left(\left(\left(\left(\left(Z_{\text {INV1 }} \| Z_{\text {Load } 1}\right)\right.\right.\right.\right.\right. \\
& \left.\left.\left.\left.\left.+Z_{\text {Line } 1}\right) \| Z_{\text {INV2 }}\right)+Z_{\text {Line } 2}\right) \| Z_{\text {Load } 2}\right)\right]^{-1}
\end{aligned}
$$

In $d-q$ domain, the basis for stability criterion is the system transfer function which is also known as the minor loop gain which is defined as follows:

$$
M L G_{d q}=\left[Z_{\text {Source }}\right]_{d q}\left[Y_{\text {Load }}\right]_{d q}
$$

The Nyquist plots for different minor loop gains (eigen loci) at different buses can be obtained by plotting roots of (34) for varied range of frequency as given by:

$$
\lambda_{d q}=\operatorname{det}\left(\lambda_{d q} I-M L G_{d q}\right)
$$

\section{A. Scenario-1: Normal operation of test microgrid}

Initially the proposed approach is applied to the test microgrid shown in Fig. 1a. There are three inverters and two loads in the microgrid. Based on the system parameters given in the appendix, an impedance model (described above) is developed under normal operating conditions to find out the strongest and weakest point in the network. Having three partition points as bus 1, 2, and 3; all the three Nyquist plots are obtained and are shown in Fig. 3a below. It can be seen from the results that the bus 2 is the most critical bus having lowest stability margin as the eigen loci is very much closer to $(-1+0 \mathrm{j})$ point. However, eigen loci at bus 3 is farthest from the $(-1+0 \mathrm{j})$ point and hence, it can be considered as the strongest point in the network from stability point of view. For quantitative analysis of nodes, gain and phase margins of the system are calculated at different partition points using Fig. 3a and are tabulated in Table I. From the Table I, it is found that bus- 2 has both lowest phase and gain margins and bus- 3 has the highest phase and gain margins. Therefore, bus- 2 is the weakest bus in the network in terms of stability margins.

Although, these results are obtained by taking droop coefficients as zero, the weakest and strongest node in the system remain the same even if the the droop values are considered. To 


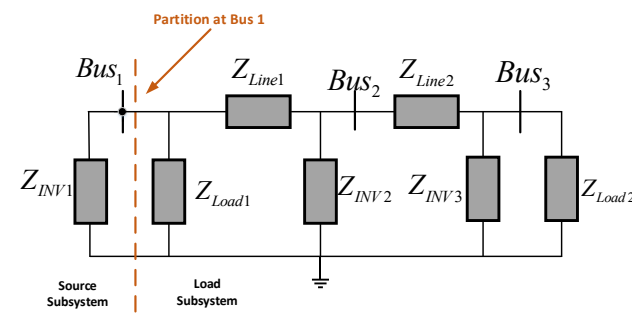

(a) Partition at bus 1

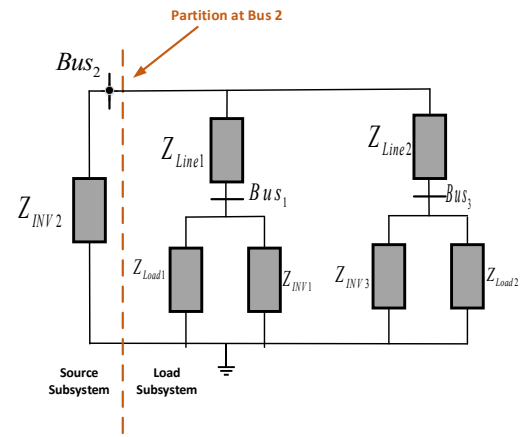

(b) Partition at bus 2

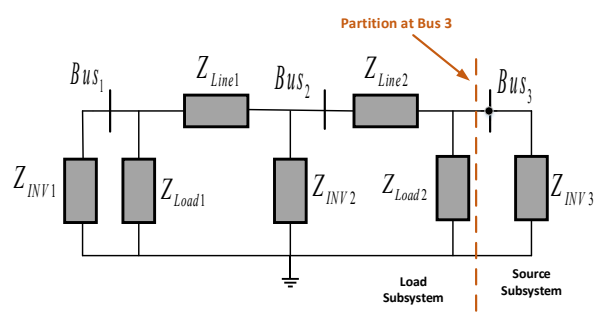

(c) Partition at bus 3

Fig. 2: Source and load equivalents at different partition points

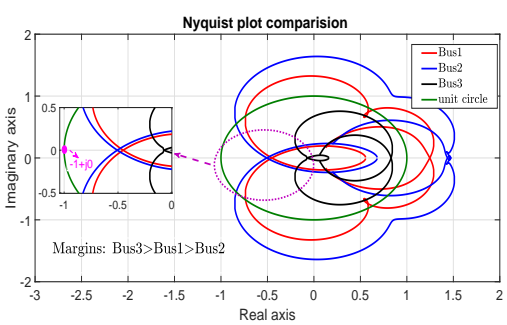

(a) Nyquist plots at different partition points

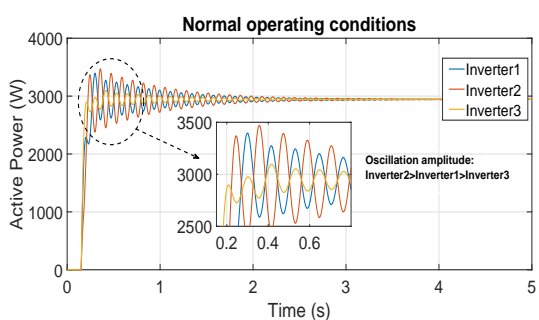

(b) Active power output of inverters

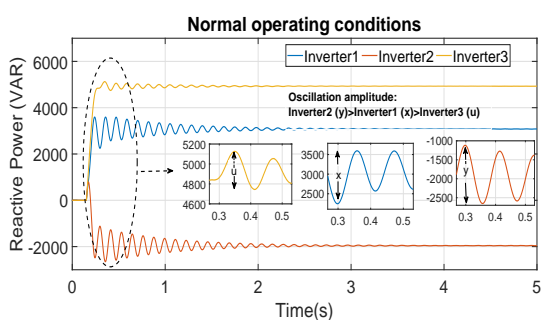

(c) Reactive power output of inverters

Fig. 3: Identification of system weakest and strongest point

verify these conclusions, time domain simulations as shown in Fig. $3 \mathrm{~b}$ are performed in which loads are turned $\mathrm{ON}$ at $\mathrm{t}=0.15$ s. The active power of all three inverters starts increasing to feed the power of load 1 and load 2 connected at bus 1 and 3 respectively. The power is oscillating in nature due to the lesser inertia and higher droop coefficient [16]. However, it can be seen that though the frequency of oscillation is same for all three inverters, the magnitude of oscillations are different in different inverters due to their location point/node in the network, since all the inverters are taken to be identical having same droop coefficients and controller parameters. The magnitude of oscillations is maximum in inverter 2 followed by inverter 1 and least in inverter 3 . This goes with the conclusion obtained from proposed impedance model that bus 2 is found to be the weakest one whereas, bus 3 is found to be the strongest one. Therefore, the proposed model is able to find out the weaknesses (stability margins) of various buses in a network. Reactive power of different inverters is also shown in Fig. 3c, where each inverter is either giving or absorbing reactive power depending upon the terminal voltage of inverter. However, frequency and magnitude of oscillations remain similar to that of active power. Hence, weakness $(W)$ of buses considering stability margins for the AC microgrid system shown in Fig. 1a are obtained as:

$$
W_{\text {bus } 2}>W_{\text {bus } 1}>W_{\text {bus } 3}
$$

Thus, it can be concluded that the order of strength of the various nodes, remains the same irrespective of the mode of interest. Further, as the active and reactive power results give similar conclusions, only the active power results are shown further in the paper and reactive power results are not shown to avoid redundancy.
The described modelling approach can be done for any type of network by representing them in equivalent impedances. The microgrid under study is the one having only three inverters, however, this method can be applied to a system having large number of inverters and buses making it useful for future design of microgrids.

\section{B. Scenario-2: Effect of adding a new load}

In this scenario, a new load is added one by one at every bus in the test microgrid. There are two loads already present at bus- 1 and bus- 3 , therefore all three inverters are giving some power initially. At $t=3.5 \mathrm{~s}$, a new load of $3 \mathrm{~kW}$ is added with the existing load one by one at each bus and the simulation results for the same are shown in Fig. 4. From the results, it is found that addition of a new load at each bus does not change the stability margins of buses. Therefore, order of weakness based on stability margins remain same as given in (36). Nyquist plots remain same as that of Fig. 3a, therefore is not shown to avoid redundancy.

\section{Scenario-3: Effect of change in line parameters}

To further verify the proposed model, parameters of line1 are changed gradually to observe its effect on stability margin of partition points. Initially parameters of line- 1 are $R=0.45 \Omega \& X=0.1 \Omega$ and parameters of line- 2 are $R=1.05 \Omega \& X=0.58 \Omega$. Resistance and reactance of line- 1 are now increased in four steps, keeping parameter of line2 constant. Nyquist contour for bus- 1 as a partition point is shown in Fig. 5a. Case-1 to case-5 describe the Nyquist plots of bus- 1 as a partition point with different values of $\mathrm{R}$ and $\mathrm{X}$ of line-1. It can be seen that on increasing the values, the Nyquist contour shifts away from $(-1+\mathrm{j} 0)$ point towards right half of the plane making the system more stable. In case- 


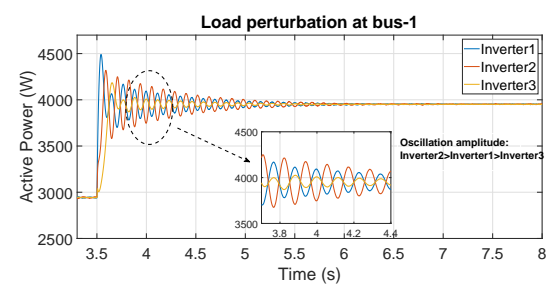

(a) Power of inverters with a new load at bus- 1

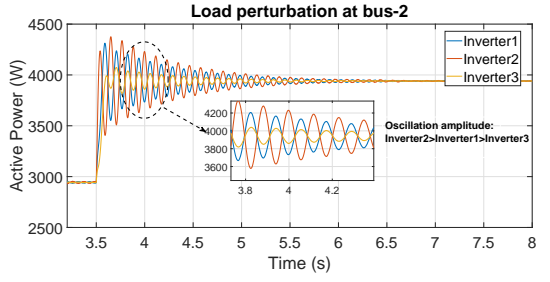

(b) Power of inverters with a new load at bus-2

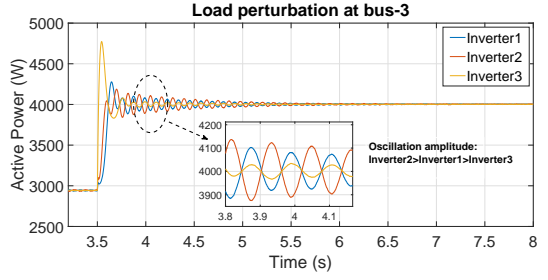

(c) Power of inverters with a new load at bus-3

Fig. 4: Effect of adding a new load

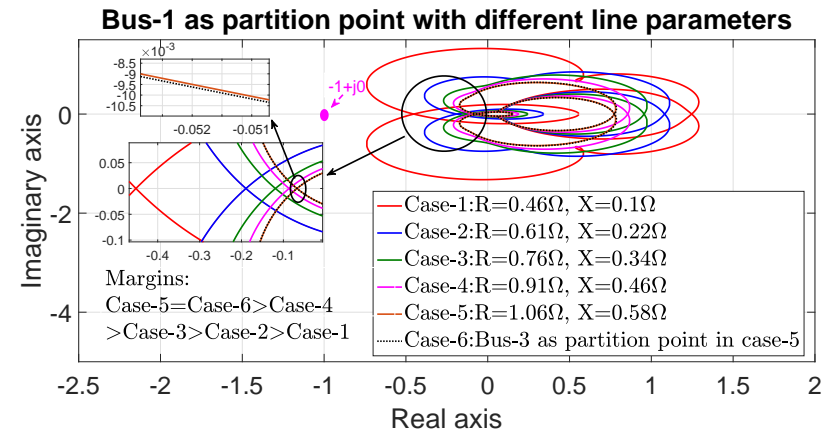

(a) Nyquist plot comparison

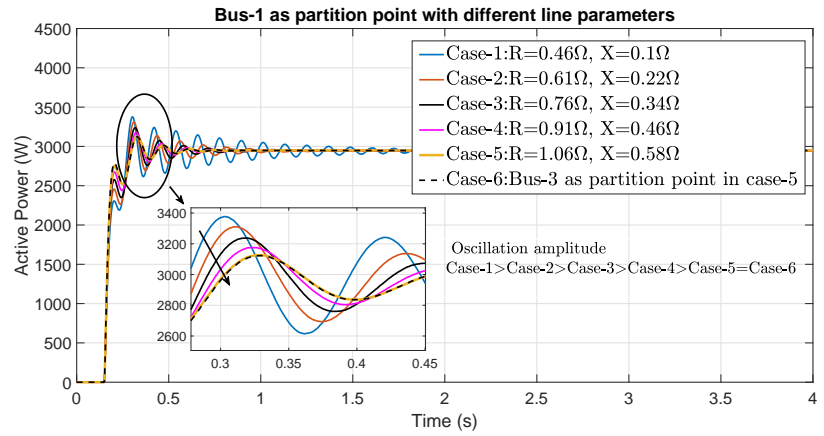

(b) Active power output of inverter-1

Fig. 5: Effect of change in line parameters

5 , parameters of line- 1 become equal to parameters of line- 2 and therefore for case-5, Nyquist contour for bus- 1 and bus-3 overlaps with each other. Nyquist plot of bus-3 as a partition point with the parameters of case- 5 is shown as case- 6 and it can be seen that case- 5 and case- 6 approximately overlap with each other making a symmetrical system about bus-2. Hence, bus-2 remains weakest due to its position in the network, but the change in stability margin is very well detected by the proposed approach. To verify the results obtained in Fig. 5a, time domain simulations are also obtained and are shown in Fig. 5b. The simulation shows the same pattern as that of Fig. 5a, as the parameters of line- 1 are increased, stability margin of bus-1 increases making the whole system more stable. Therefore, it is found that changing network parameters changes the weakness of points in a network which can be accurately determined by the proposed approach.

\section{Scenario-4: Effect of change of load location}

In this scenario, the location of existing load 1 and load 2

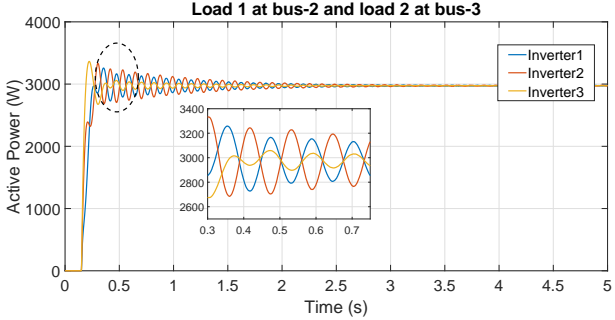

(a) Active power output of inverters

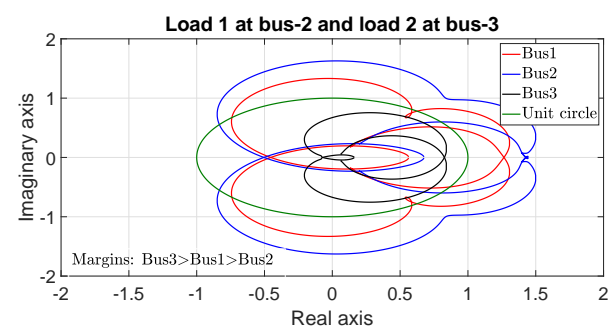

(b) Nyquist plots at different partition points

Fig. 6: Effect of load location (load 1 at bus-2 and load 2 at bus-3)

is changed to observe the effect of load location on strongest and weakest point. Initially in scenario 1 , load 1 is placed at bus- 1 and load 2 is placed at bus-3. Here, we take two other cases as follows:

(a) When load 1 is at bus- 2 and load 2 is at bus-3: When the location of load changes, then at the instance of switching ON the loads, the initial peak in inverter power may vary depending on which inverter is nearer to load. However, from the Fig. 6a, it is found that subsequent oscillations follow the same pattern as that of scenario-1. Therefore, bus2 remains the weakest and bus- 3 remains strongest in terms of stability margins, irrespective of load location. Similarly, Nyquist contours are also similar to those of scenario- 1 and are shown in Fig. 6 b.

(b) When load 1 is at bus-2 and load 2 is at bus-1: In this case, load 1 is shifted to bus- 2 and load 2 is shifted to bus- 1 in the test microgrid. As soon as the loads are turned ON at $\mathrm{t}=0.15 \mathrm{~s}$, oscillations appear due to the inherent nature of droop controlled microgrids as shown in Fig. 7a. However, in this case also magnitude of oscillations follow the same pattern as that of case(a). Still maximum margins are found at bus-3 and minimum at bus- 2 and therefore, Nyquist plots remain similar 


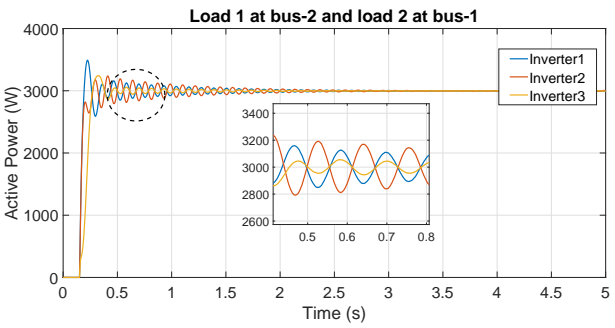

(a) Active power output of inverters

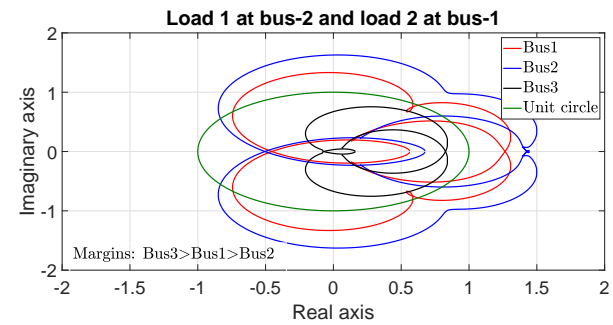

(b) Nyquist plots at different partition points

Fig. 7: Effect of load location (load 1 at bus-2 and load 2 at bus-1)

and are shown in Fig. $7 \mathrm{~b}$.

\section{E. Scenario-5: Sensitivity to parameter variation}

To analyze the sensitivity to small variations in system parameters, the parameters of line- 1 and line- 2 are changed as $\pm 10 \%$ and the Nyquist contours are drawn to observe the effect. First, parameters of line- 1 and line- 2 are increased by $10 \%$ and Nyquist contours at different partition points are shown in Fig. 8a. After this, the parameters of line- 1 and line- 2 are decreased by $10 \%$ and Nyquist contours at different partition points are shown in Fig. 8b. From both the results it is found that within reasonable variation in line parameters, the conclusions drawn from the proposed model remain same. Therefore, weakest bus is bus- 2 and the strongest one is bus- 3 . In other words, largest stability margins are found at bus- 3 and lowest at bus-2. This concludes that the analysis presented in this paper will give acceptable results even if there is nominal difference between the actual and the considered parameters, even though there may be slight variations in the Nyquist contours obtained in these cases.

\section{Stability Acquainted Design and Planning of NETWORK}

This section shows the application of identified weakest and strongest points in a network for planning purpose. When a new component is going to be connected to the existing network, the overall stability of the system must remain intact. To ensure the sufficient stability margins, the damping ratios of different modes must remain in acceptable limits. Identified weakest and strongest points will help a design engineer for most stable design of microgrid system. To illustrate this, first overall stability of microgrid is to be determined, which is done by state space modelling based stability analysis.

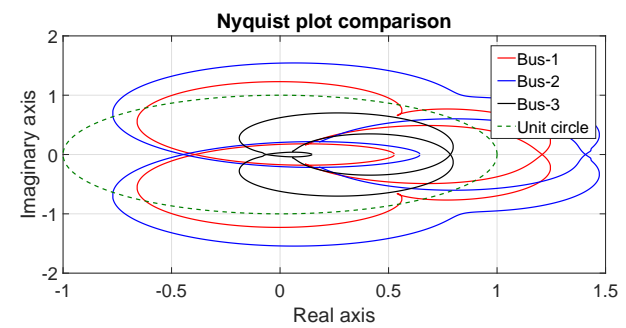

(a) $10 \%$ increase in line parameters

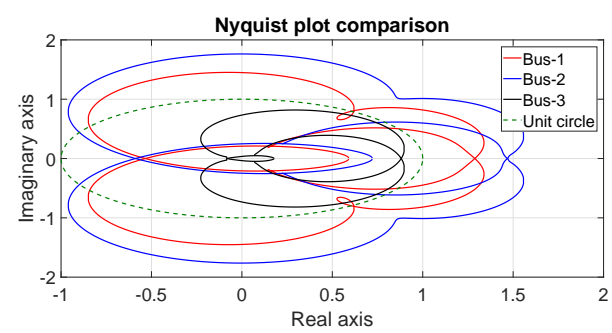

(b) $10 \%$ decrease in line parameters

Fig. 8: Sensitivity to parameter variation

\section{A. Stability analysis of complete microgrid system}

Eigen value analysis based approach is used which is based on calculating system state matrix from state space representation. Therefore, complete microgrid system must be represented in state space form as given by (37). This can be obtained by representing equations of droop, voltage and current controllers ((1)-(15)) along with equations of coupling branch, connecting lines and loads ((23)-(25)) in state space form and then combining all of them on a common frame of reference.

$$
\dot{x}(t)=A x(t)+B u(t)
$$

Any inverter frequency can be used as common reference frame. An angle $\delta$ defined by (38) is used to convert variables from respective frame of references to common reference and vise versa using (39).

$$
\delta=\int\left(\omega-\omega_{r e f}\right) d t
$$

Therefore, there is an extra state equation, considering $\delta$ as state variable, corresponding to the droop controller of each inverter. Output variables of inverter should be converted to common reference frame using the transformation given by (39) and input variables of inverter should also be converted to common through reverse transformation.

$$
f_{D Q}=T_{i} f_{d q}
$$

where

$$
T_{i}=\left[\begin{array}{cc}
\cos \left(\delta_{i}\right) & -\sin \left(\delta_{i}\right) \\
\sin \left(\delta_{i}\right) & \cos \left(\delta_{i}\right)
\end{array}\right]
$$

There are two intermediate states corresponding to PI regulator of voltage and current controller respectively as given by:

$$
\frac{d \phi_{d q}}{d t}=v_{o d q}{ }^{*}-v_{o d q} \quad ; \frac{d \gamma_{d q}}{d t}=i_{l d q}{ }^{*}-i_{l d q}
$$

The bus voltages in a AC microgrid is defined by (41), where $i_{o D Q i}$ denotes the injection at bus and $R_{N}$ is assumed 


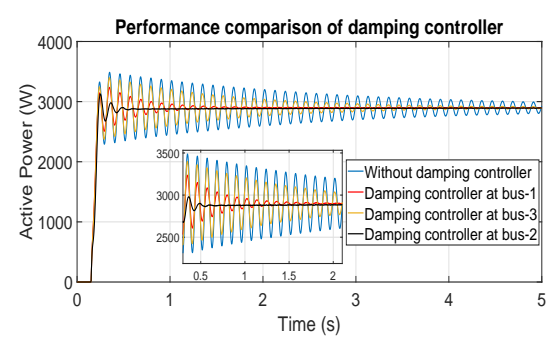

(a) On addition of damping controller

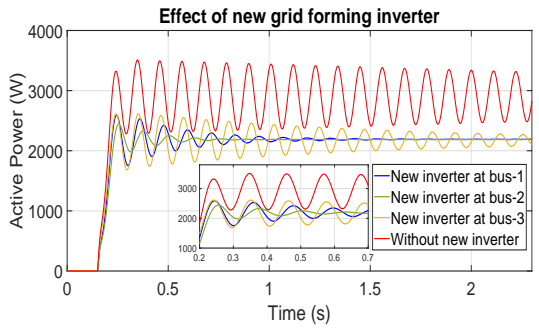

(b) On addition of grid forming inverter

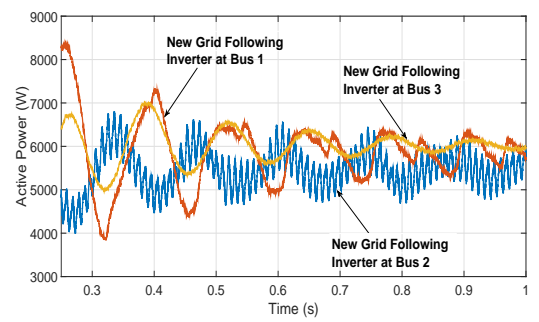

(c) On addition of grid feeding inverter

Fig. 9: Power of inverter 2 on addition of different components

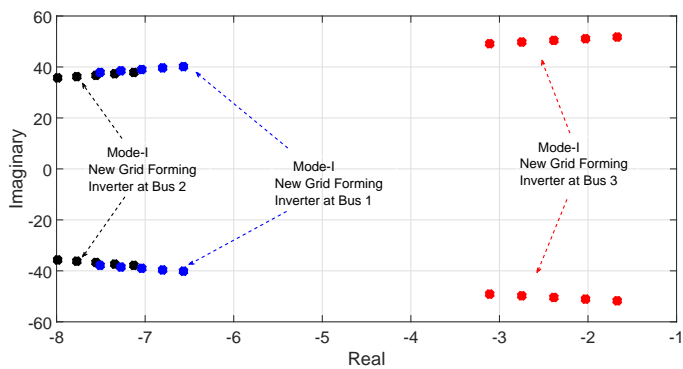

(a) With the increase in $m$ of grid forming inverter

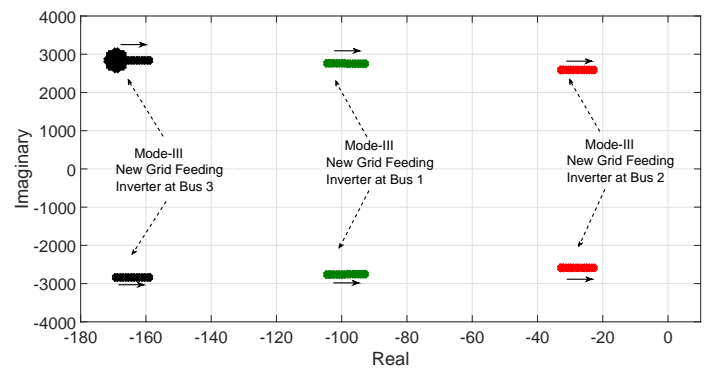

(b) With the increase in $K_{i p}$ of grid feeding inverter

Fig. 10: Dominant eigen value trace when adding a new inverter in grid forming and grid feeding modes

TABLE II: Dominant Low Frequency Modes

\begin{tabular}{|c|c|c|}
\hline No. of Modes & Eigen Value & Damping Ratio $(\zeta \%)$ \\
\hline Mode-I & $-0.2217 \pm 46.64 \mathrm{i}$ & 0.47 \\
\hline Mode-II & $-12.96 \pm 28.64 \mathrm{i}$ & 41.2 \\
\hline
\end{tabular}

a sufficiently large passive resistance which has minimum contribution in dynamic stability of the system.

$$
v_{b D Q i}=R_{N}\left(i_{o D Q i}-i_{\text {LoadDQ }} \pm i_{\text {LineDQi }}\right)
$$

Combining all dynamical equations (1)-(15), (23)-(25), (37)-(40), on a common reference will lead to the state equation of overall microgrid given as:

$$
\left[\begin{array}{c}
\Delta \dot{x}_{\text {inv }} \\
\Delta i_{\text {linedq }} \\
\Delta i_{\text {loaddq }}
\end{array}\right]=A_{m g}\left[\begin{array}{c}
\Delta x_{\text {inv }} \\
\Delta i_{\text {linedq }} \\
\Delta i_{\text {loaddq }}
\end{array}\right]
$$

The state vector contains total 47 states (39 (inverter), 4 (load), 4 (lines)). From stability analysis, eigen values are calculated using $A_{m g}$. It consists of three clusters: high, medium, and low frequency modes out of which high and medium frequency modes are much stable than the low frequency modes. There are two critical low frequency modes which are sensitive to droop control of inverter [16]; one among them having very low damping ratio $(\zeta)$ as shown in Table II and is responsible for determining system stability. Therefore, design and planning studies should consider damping of these critical modes which will be illustrated subsequently.

\section{B. Application of critical points for different scenarios}

1) Suitable placement of damping controllers: As mentioned in the previous section, parallel operation of grid- forming inverters causes lightly damped low frequency oscillations which can cause instability of the system. To suppress such type of oscillations, many damping controllers have been developed in literature [14]-[18]. One of them is based on decentralised lead compensator as developed in [16]. The dynamic equation for the same is given in (43) and (44). In [16], the damping controller is placed at each and every inverter present in a system. However, suitable location must be identified for cost effective operation since it is not economical to modify each and every inverter control. For the same, usually optimization techniques are used which is based on maximizing the damping of critical modes using eigen values. In this regard, critical point obtained from the impedance based analysis explained in section-IV can be used for suitable location of damping controllers.

$$
\begin{gathered}
P_{1}=p * K *\left(\frac{1+s T_{1}}{1+s T_{2}}\right) \\
\Delta \omega_{P S S}=\left(\frac{s}{1+s T_{w}}\right) * P_{1}
\end{gathered}
$$

Stability margins and critical modes obtained when a damping controller is placed one by one at each inverter is tabulated in Table III. It can be seen that maximum stability margin with improved damping ratio can be achieved when the damping controller is placed at inverter on bus no. 2 which is found to be the most critical bus for a system shown in Fig. 1a. However, when the controller is placed on bus no. 3, which is the strongest point in the network, lowest stability margin is obtained. This can also be verified from the simulation results shown in Fig. 9a. The low frequency oscillations are relatively more damped when controller is placed at inverter no. 2. This is due to the fact that by adding the damping controller at the weakest node, there is maximum potential for 
TABLE III: Relative Stability Margins on Addition of Damping Controllers

\begin{tabular}{|c|c|c|c|}
\hline Location of damping Controller & Critical Droop Value & Critical Mode at m=2.6 $\times 10^{-4}$ & Damping Ratio $(\zeta \%)$ \\
\hline No damping controller & $2.6 \times 10^{-4}$ & $-0.2217 \pm 46.64 \mathrm{i}$ & 0.47 \\
\hline Damping controller at Inverter 1 & $10.6 \times 10^{-4}$ & $-6.00 \pm 43.25 \mathrm{i}$ & 13.74 \\
\hline Damping controller at Inverter 2 & $22 \times 10^{-4}$ & $-11.82 \pm 38.62 \mathrm{i}$ & 29.26 \\
\hline Damping controller at Inverter 3 & $4.3 \times 10^{-4}$ & $-1.38 \pm 45.92 \mathrm{i}$ & 3.01 \\
\hline
\end{tabular}

improving the overall system stability. On the contrary, if the damping controller is added to the strongest point, it doesn't have much scope of improvement as it is already stable and the system's weakest point still remains the main source of instability. Hence, for planning purpose, it is always advisable to put compensator on the most critical bus rather than the strongest bus.

2) Addition of a new grid forming inverter: If the new inverter which is going to be connected at any bus in a microgrid is a grid forming one, it has the same control (Fig. 1b) as that of existing inverters. Then the impedance at the bus where it is connected will get halved which will lead to the higher stability margins. This implies that a grid forming inverter will help in stability improvement for an existing grid forming inverter. However, it's impact will be different at different buses similar to the case of damping controller. So, optimum location needs to be found out in this case as well. For this purpose, overall system state matrix is again calculated one by one having fourth inverter at each bus. Total number of states is increased by 13 and now the order of state matrix is $(60 \times 60)$. The damping ratios $(\zeta)$ of critical modes in all the three cases is summarized in Table IV. In Table IV, only the variation of overall effect of new inverters on system stability is shown while the stability analysis of these new inverters is well reported in literature and it is not shown here due to avoid redundancy.

It is found that, due to the addition of a new droop controlled inverter, a new low frequency oscillation corresponding to the droop controller of fourth inverter, gets added to the system (Mode III) which is independent of the point of connection. However, it improves the transient response of existing system by providing damping to the existing critical mode (Mode I and Mode II). Since, it is acting as a stability enhancing component like the damping controller discussed in previous section, it provides maximum damping when connected to the weakest or most critical node. This can also be verified through simulation results shown in Fig. $9 \mathrm{~b}$ and the eigen value trace shown in Fig. 10a. It can be seen that when the new inverter is added at the weakest bus, the trace of eigen value shifts to the maximum left while it shifts the minimum when the new inverter is added to the strongest bus. So, we can conclude that the ideal location for adding new inverter is the weakest bus of the system.

3) Addition of a new grid feeding inverter: Suppose the new inverter which is going to be connected to droop controlled microgrid is grid feeding type, then the control loop of new inverter changes and the new control is shown in Fig. 11.

To investigate the effect of new grid feeding inverter on droop controlled microgrid, overall state matrix is again calculated having new dynamical equations of grid feeding inverter defined as follows: The droop frequency equation is

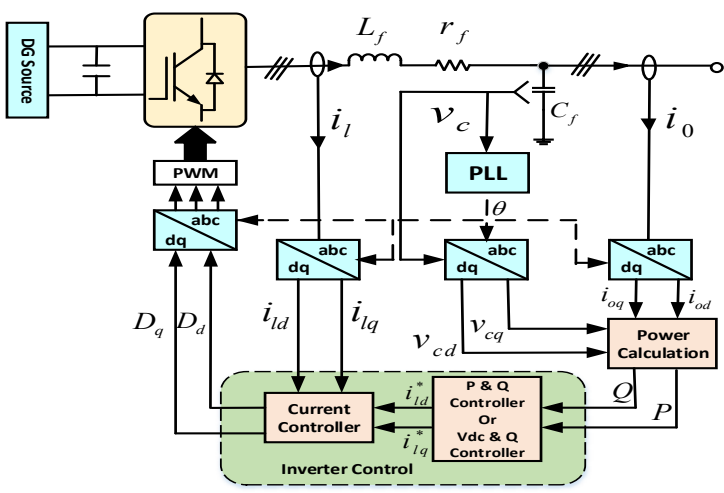

Fig. 11: Control diagram of a grid feeding inverter

not applicable for the new inverter, hence, it requires PLL to generate its $\theta$ for $d-q$ transformation. The PLL working is based on aligning the angle of $d q$ transformation so as to get $v_{c q}=0$ at the connection point. A PI controller is used to act on alignment error to set the angular frequency which is further used to generate angle.

$$
\begin{gathered}
\omega=K_{p}^{P L L} v_{c q}+K_{i}^{P L L} \int v_{c q} d t \\
\theta=\int \omega d t
\end{gathered}
$$

Inner current controller is same as that of grid forming inverter, but outer loop control changes for grid feeding one as given by:

$$
\begin{gathered}
\frac{d \phi_{d q}}{d t}=P_{r e f}{ }^{*}-P \quad ; \frac{d \gamma_{d q}}{d t}=Q_{r e f}{ }^{*}-Q \\
i_{l d}^{*}=\left(K_{p p}+K_{i p} / s\right)\left(P_{r e f}{ }^{*}-P\right) \\
i_{l q}^{*}=\left(K_{p p}+K_{i p} / s\right)\left(Q_{r e f}{ }^{*}-Q\right) \\
v_{i d}^{*}=-\omega L_{f} i_{l q}+\left(K_{p}+K_{i} / s\right)\left(i_{l d}^{*}-i_{l d}\right)+v_{c d} \\
v_{i q}^{*}=\omega L_{f} i_{l d}+\left(K_{p}+K_{i} / s\right)\left(i_{l q}^{*}-i_{l q}\right)+v_{c q}
\end{gathered}
$$

$P_{r e f}{ }^{*}$ and $Q_{r e f}{ }^{*}$ are defined as per the requirement of the network. $P$ and $Q$ are calculated with the terminal measurements as given in (1)-(4). In this work, $Q_{r e f}{ }^{*}$ is set to be zero for unity power factor operation. Filter and coupling branch dynamic equations remain same as given by (12)-(15). Now the overall state matrix of the microgrid with new grid feeding inverter can be obtained by linearising and solving (12)-(15), (23)-(25). and (45)-(50) on a common reference frame and combining them with the microgrid state space model (42). Number of states are increased by 12 and order of system matrix becomes $(59 \times 59)$. Eigen value can be obtained using system state matrix. Critical modes are shown in the Table IV.

From the Table IV, it is found that the new grid feeding inverter does not interact much with the low frequency oscillation of droop controlled microgrid (Mode I and Mode 
TABLE IV: Relative Stability Margins with the Addition of New Inverter

\begin{tabular}{|c|c|c|}
\hline \multicolumn{3}{|c|}{ Addition of New Grid Forming Inverter } \\
\hline At Bus 1 & At Bus 2 & At Bus 3 \\
\hline Mode $\mathrm{I}=-7.98 \pm 36.71 j(\zeta=21.2 \%)$ & Mode $\mathrm{I}=-9.98 \pm 32.92 j(\zeta=29.3 \%)$ & Mode $\mathrm{I}=-4.31 \pm 41.67 j(\zeta=10.3 \%)$ \\
\hline Mode $\mathrm{II}=-14.96 \pm 14.05 j(\zeta=72.6 \%)$ & Mode $\mathrm{II}=-14.96 \pm 14.47 j(\zeta=71.8 \%)$ & Mode $\mathrm{II}=-15.11 \pm 13.90 j(\zeta=73.4 \%)$ \\
\hline Mode $\mathrm{III}=-18.83 \pm 63.93 j(\zeta=28.25 \%)$ & Mode $\mathrm{III}=-18.83 \pm 63.93 j(\zeta=28.25 \%)$ & Mode $\mathrm{III}=-18.83 \pm 63.93 j(\zeta=28.25 \%)$ \\
\hline \multicolumn{3}{|c|}{ Addition of New Grid Feeding Inverter } \\
\hline At Bus 1 & At Bus 2 & At Bus 3 \\
\hline Mode $\mathrm{I}=-0.88 \pm 48.68 j(\zeta=1.8 \%)$ & Mode $\mathrm{I}=-0.79 \pm 48.02 j(\zeta=1.6 \%)$ & Mode $\mathrm{I}=-0.93 \pm 47.92 j(\zeta=1.9 \%)$ \\
\hline Mode $\mathrm{II}=-13.19 \pm 28.50 j(\zeta=42 \%)$ & Mode $\mathrm{II}=-13.02 \pm 28.07 j(\zeta=42.07 \%)$ & Mode $\mathrm{II}=-13.46 \pm 28.80 j(\zeta=42.3 \%)$ \\
\hline Mode III $=-78.84 \pm 2615.8 j(\zeta=3.1 \%)$ & Mode III $=-32.54 \pm 2842.1 j(\zeta=1.1 \%)$ & Mode $\mathrm{III}=-169.08 \pm 2521.3 j(\zeta=9.9 \%)$ \\
\hline
\end{tabular}

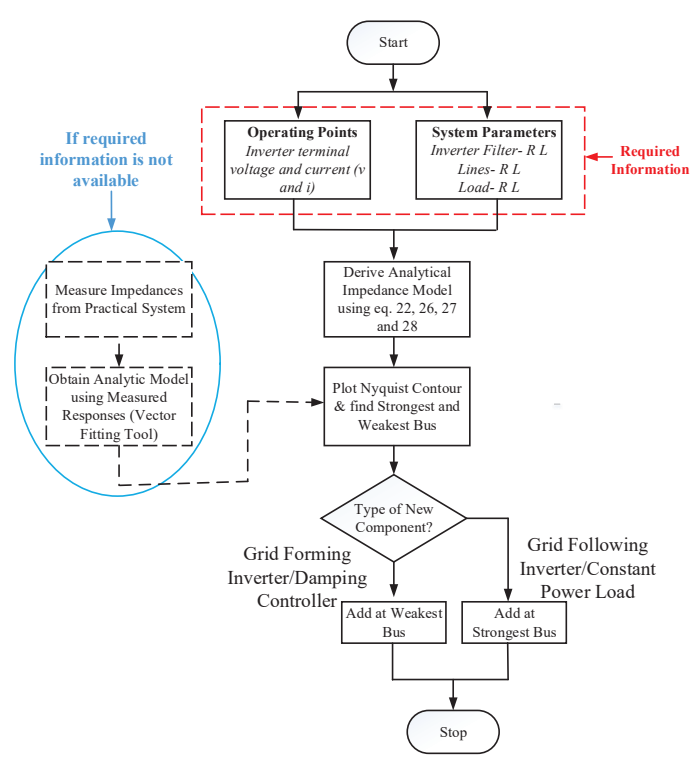

Fig. 12: Flowchart for proposed design of microgrid

II). However, a new medium frequency mode (Mode III) gets excited which can reduce stability margins of the microgrid. It can also be concluded from the results that damping of this medium frequency mode also changes with the location of connection. Since, the nature of grid feeding inverter is harmful for medium frequency stability [9], [30], it degrades the stability margin most when connected to the already weakest node, i.e. bus no. 2. Time domain simulation results shown in Fig. 9c also show that a medium frequency mode loses its damping on addition of a new grid feeding inverter while there is not much change in low frequency oscillation. This is due to the fact that a weak node will become even weaker due to negative incremental resistance (due to constant power mode of operation [9]) provided from the grid feeding inverter. On the contrary, it provides much larger stability margin when connected to the strongest node in a network i.e. bus no.3. This is also verified from the trace of eigen values shown in Fig. 10b, where it can be clearly seen that for the medium frequency modes, the eigen values are to the maximum left when the new inverter is connected to bus 3. In this way, stability oriented planning for expansion of microgrids can be well made.

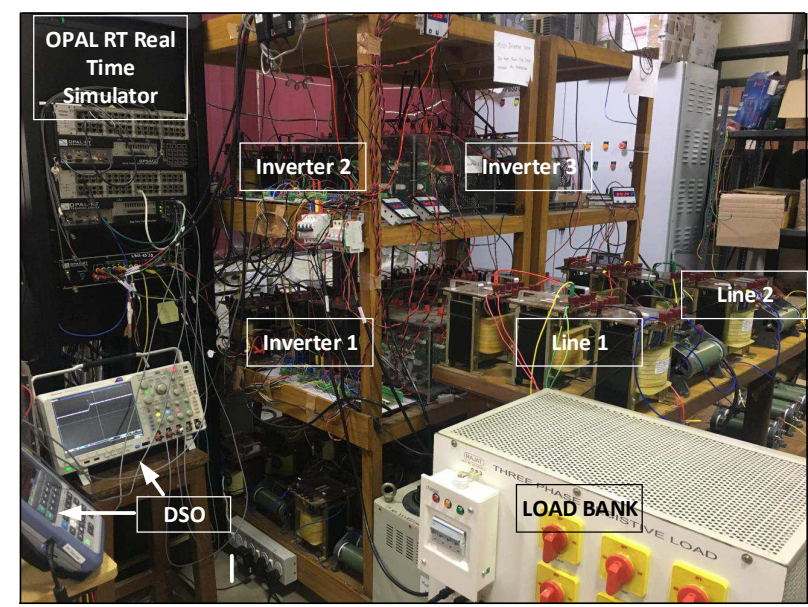

Fig. 13: Hardware Prototype [19]

\section{Summary and discussion}

This method is suitable for off-line planning of network expansion. Usually, we have already acquired some knowledge of the system which can help us fulfil the presented modelling and analysis. Even if configurations change constantly, the speed of change is not defying the method, since the speed of change is not that fast. Moreover, the method can also be used in the design stage of a new microgrid for knowing a priori the weakest/strongest points for VSI/CSI connection, and even to perhaps avoid weakest points and design microgrids with stronger points. However, in the future, if online planning is focused and if some of the parameters are not known, then the impedance identification should be incorporated in the current framework. This can be achieved by combining the following two techniques: 1) impedance measurement (getting the response of a component from a practical system), 2) The vector-fitting tool (to generate the analytic model from the measured responses [38], [39]). The complete procedure is shown in Fig. 12.

Further, the conclusions of proposed scheme doesn't indicate that if a new distributed generation is connected at any bus randomly, then the whole system will get unstable (Table III and IV). A new inverter can be added to any bus and the system may remain stable. It is only the design perspective that if the source is connected at a specific bus, as obtained from the proposed algorithm, the stability margins of the microgrid get increased. Therefore, the proposed algorithm provides information about the connecting points that if used 


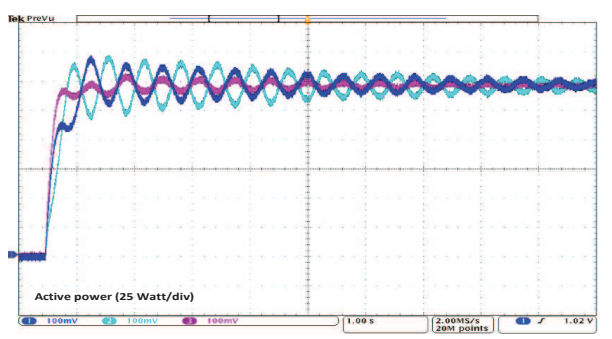

(a) Active power

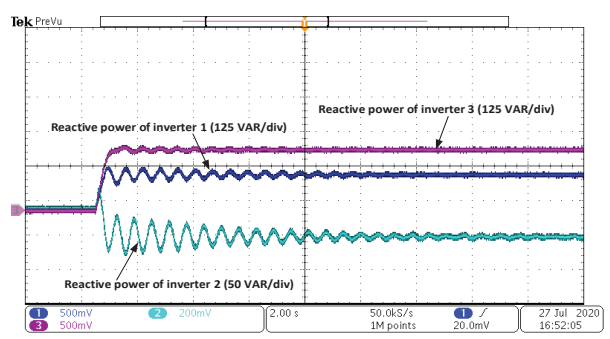

(b) Reactive power

Fig. 14: Active and reactive power output without damping controller

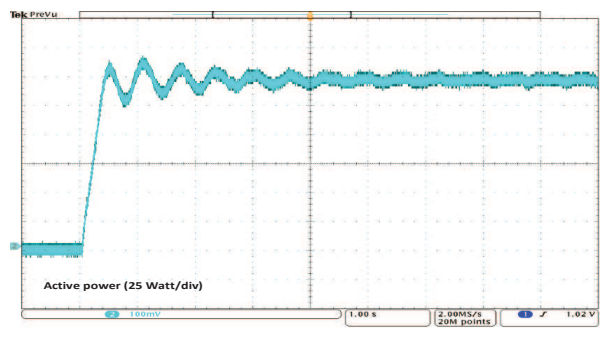

(a) When damping controller is placed at bus-1

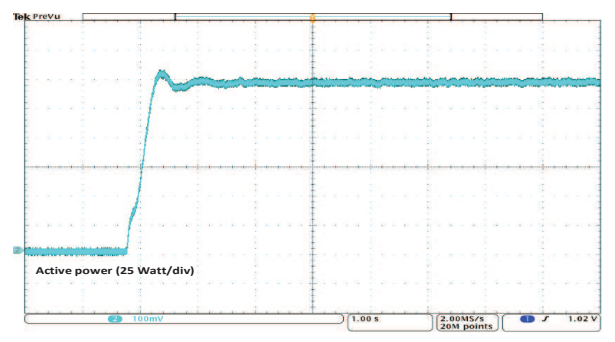

(b) When damping controller is placed at bus-2

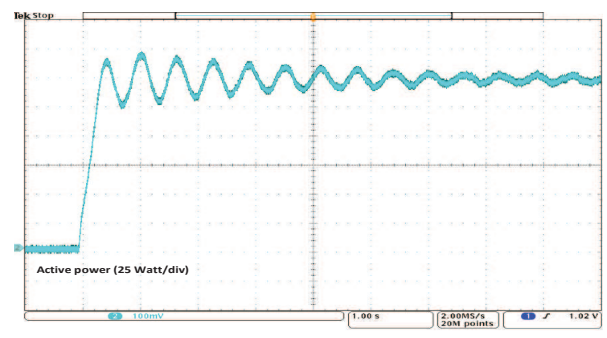

(c) When damping controller is placed at bus-3

Fig. 15: Active power output of inverter 2

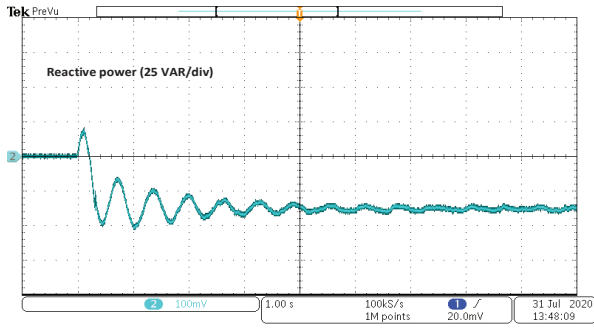

(a) When damping controller is placed at bus-1

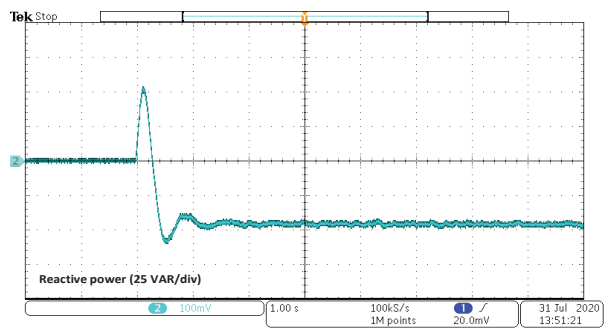

(b) When damping controller is placed at bus-2

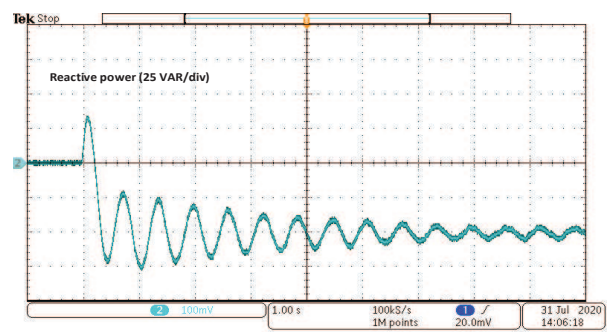

(c) When damping controller is placed at bus-3

Fig. 16: Reactive power output of inverter 2

will provide the system with higher stability margins.

\section{EXPERIMENTAL RESUlTS}

To verify the findings obtained from the proposed algorithm, a three inverter setup (as per test microgrid) is developed and tested in the laboratory as shown in Fig. 13. The experimental validation of the test system has also been done in [19]. The firing PWM gate signal for inverters are generated in OPALRT real time simulator by simulating inverter control in it. The responses for active and reactive powers of inverter are shown for verification.

Initially, when there is no damping controller in inverters, as soon as load is switched ON, all three inverters start oscillating as shown in Fig. 14a and Fig. 14b. It can be seen from the results that the magnitude of oscillations seen follow the same nature as obtained in simulation results. Further, to validate the performance of damping controller at different location, it is placed one by one at different inverters and the results are shown in Fig. 15 and Fig. 16. Results are shown only for inverter 2 in order to maintain the clarity of the results. It is found that if the damping controller is placed on the weakest node as obtained earlier, it provides maximum damping to the system oscillations. Hence, the system configuration obtained by the proposed algorithm is experimentally validated.

\section{CONCLUSION}

This paper is divided into two parts. The first part shows the application of the impedance-based method to identify the weakest and the strongest nodes in terms of stability margins in a droop controlled AC microgrid. The second part investigates the effect of adding a new inverter in existing droop controlled microgrid. From the results obtained, it is found that having a knowledge of the weakest and strongest point in a droop controlled microgrid (as obtained in the first part) will be helpful in the stability-oriented design of the network. If the new grid forming inverter is going to be connected to an existing droop controlled microgrid, it should be added to the weakest point of the network. However, if the new inverter is grid feeding one, it should be added to the strongest point in the network. These results are verified using eigenvalue 
analysis and time-domain simulations. Moreover, the suitable location of damping controllers can also be decided using the knowledge of the weakest point. This paper, thus, gives a method to select ideal locations for expansion and design of microgrid to ensure maximum stability margins.

\section{APPENDIX}

\section{A. System parameters}

TABLE V: Test Microgrid Parameters

\begin{tabular}{c}
\hline \hline$\omega_{c}=31.41 \mathrm{rad} / \mathrm{sec} ; V_{n}=311 \mathrm{~V} ; \omega_{n}=314 \mathrm{rad} / \mathrm{sec} ; K_{i v}=13.5716 ; F=0.75 ;$ \\
$L_{f}=1.35 \mathrm{mH} ; C_{f}=50 \mu \mathrm{F} ; r_{f}=0.1 \Omega ; L_{c}=0.35 \mathrm{mH} ; r_{c}=0.03 \Omega ;$ \\
$K_{p v}=0.1682 ; K_{i v}=189.345 ; m=8 \mathrm{e}-5 ; n=1.3 \mathrm{e}-5 ; K_{i i}=1005.310 ;$ \\
$L_{L i n e 1}=0.383 m H ; R_{\text {Line } 1}=0.46 \Omega ; L_{L i n e 2}=1.846 m H ;$ \\
$R_{\text {Line } 2}=1.05 \Omega ; X_{\text {Load } 1}=15.7 \Omega ; R_{L o a d 1}=20 \Omega ;$ \\
$X_{\text {Load } 1}=14.5 \Omega ; R_{\text {Load } 2}=25 \Omega ; T_{w}=3 ; T_{1}=0.47 ; T_{2}=0.01 ;$ \\
$K=5 \mathrm{e}-5 ; K_{p p}=0.1682 ; K_{i p}=15.2 ; K_{p}^{P L}=180 ; K_{i}^{P L L}=3200$ \\
\hline \hline
\end{tabular}

\section{B. Source Impedance of Grid Forming Inverter}

$$
\begin{aligned}
& {[K 1]=\left[\begin{array}{cc}
\left(R_{f}+s L_{f}+G_{i}(s)\right) / G_{i}(s) & 0 \\
0 & \left(R_{f}+s L_{f}+G_{i}(s)\right) / G_{i}(s)
\end{array}\right]} \\
& {[K 2]=\left[\begin{array}{ll}
-i_{l q}^{o} L_{f} / G_{i}(s) & 0 \\
-i_{l d}^{o} L_{f} / G_{i}(s) & 0
\end{array}\right] ; \quad[K 3]=\left[\begin{array}{cc}
-G_{v}(s) & -\omega^{o} C_{f} \\
\omega^{o} C_{f} & -G_{v}(s)
\end{array}\right]} \\
& {[K 4]=\left[\begin{array}{cc}
H & 0 \\
0 & H
\end{array}\right] ; \quad[K 5]=\left[\begin{array}{cc}
0 & G_{v}(s) \\
0 & 0
\end{array}\right] ; \quad[K 6]=\left[\begin{array}{cc}
1 & 0 \\
0 & 1
\end{array}\right]} \\
& {[K 7]=\left[\begin{array}{cc}
s C_{f} & -\omega^{o} C_{f} \\
\omega^{o} C_{f} & s C_{f}
\end{array}\right] ; \quad[K 8]=\left[\begin{array}{cc}
0 & 0 \\
v_{c d}^{o} C_{f} & 0
\end{array}\right]} \\
& {[K 9]=\left[\begin{array}{ll}
-1.5 m G_{L P}(s) i_{o d}^{o} & -1.5 m G_{L P}(s) i_{o q}^{o} \\
-1.5 n G_{L P}(s) i_{o q}^{o} & 1.5 n G_{L P}(s) i_{o d}^{o}
\end{array}\right]} \\
& {[K 10]=\left[\begin{array}{cc}
-1.5 m G_{L P}(s) v_{c q}^{o} & 0 \\
0 & -1.5 n G_{L P}(s) v_{c d}^{o}
\end{array}\right]} \\
& {[K i]=[K 1][K 8][k 10]+[K 2][K 10]-[K 5][K 10]-[K 4]+[K 1][K 6]} \\
& {[J i]=[K 3]-[K 1][K 7]-[K 1][K 8][k 9]-[K 2][K 9]+[K 5][K 9]}
\end{aligned}
$$

\section{REFERENCES}

[1] M. C. Chandorkar, D. M. Divan, and R. Adapa, "Control of parallel connected inverters in standalone ac supply systems," IEEE Transactions on Industry Applications, vol. 29, no. 1, pp. 136-143, Jan 1993.

[2] E. A. A. Coelho, P. C. Cortizo, and P. F. D. Garcia, "Small-signal stability for parallel-connected inverters in stand-alone ac supply systems," IEEE Transactions on Industry Applications, vol. 38, no. 2, pp. 533-542, Mar 2002.

[3] N. Pogaku, M. Prodanovic, and T. C. Green, "Modeling, analysis and testing of autonomous operation of an inverter-based microgrid," IEEE Transactions on Power Electronics, vol. 22, no. 2, pp. 613-625, March 2007.

[4] Y. Pan, L. Chen, X. Lu, J. Wang, F. Liu, and S. Mei, "Stability region of droop-controlled distributed generation in autonomous microgrids," IEEE Transactions on Smart Grid, vol. 10, no. 2, pp. 2288-2300, March 2019.

[5] A. S. Vijay, D. K. Dheer, A. Tiwari, and S. Doolla, "Performance evaluation of homogeneous and heterogeneous droop-based systems in microgridstability and transient response perspective," IEEE Transactions on Energy Conversion, vol. 34, no. 1, pp. 36-46, March 2019.

[6] G. Raman and J. C. Peng, "Mitigating stability issues due to line dynamics in droop-controlled multi-inverter systems," IEEE Transactions on Power Systems, vol. 35, no. 3, pp. 2082-2092, 2020.

[7] A. Kahrobaeian and Y. A. R. I. Mohamed, "Analysis and mitigation of low-frequency instabilities in autonomous medium-voltage converterbased microgrids with dynamic loads," IEEE Transactions on Industrial Electronics, vol. 61, no. 4, pp. 1643-1658, April 2014.

[8] A. A. A. Radwan and Y. A. I. Mohamed, "Stabilization of mediumfrequency modes in isolated microgrids supplying direct online induction motor loads," IEEE Transactions on Smart Grid, vol. 5, no. 1, pp. 358370, Jan 2014.
[9] N. Bottrell, M. Prodanovic, and T. C. Green, "Dynamic stability of a microgrid with an active load," IEEE Transactions on Power Electronics, vol. 28, no. 11, pp. 5107-5119, Nov 2013.

[10] J. Chen and J. Chen, "Stability analysis and parameters optimization of islanded microgrid with both ideal and dynamic constant power loads," IEEE Transactions on Industrial Electronics, vol. 65, no. 4, pp. 3263 3274, April 2018.

[11] M. A. Hassan, "Dynamic stability of an autonomous microgrid considering active load impact with a new dedicated synchronization scheme," IEEE Transactions on Power Systems, vol. 33, no. 5, pp. 4994-5005, Sep. 2018.

[12] K. Yu, Q. Ai, S. Wang, J. Ni, and T. Lv, "Analysis and optimization of droop controller for microgrid system based on small-signal dynamic model," IEEE Transactions on Smart Grid, vol. 7, no. 2, pp. 695-705, March 2016.

[13] M. A. Hassan and M. A. Abido, "Optimal design of microgrids in autonomous and grid-connected modes using particle swarm optimization," IEEE Transactions on Power Electronics, vol. 26, no. 3, pp. 755-769, March 2011.

[14] Y. A. R. I. Mohamed and E. F. El-Saadany, "Adaptive decentralized droop controller to preserve power sharing stability of paralleled inverters in distributed generation microgrids," IEEE Transactions on Power Electronics, vol. 23, no. 6, pp. 2806-2816, Nov 2008.

[15] R. Majumder, B. Chaudhuri, A. Ghosh, R. Majumder, G. Ledwich, and F. Zare, "Improvement of stability and load sharing in an autonomous microgrid using supplementary droop control loop," IEEE Transactions on Power Systems, vol. 25, no. 2, pp. 796-808, May 2010.

[16] A. Firdaus and S. Mishra, "Mitigation of power and frequency instability to improve load sharing among distributed inverters in microgrid systems," IEEE Systems Journal, vol. 14, no. 1, pp. 1024-1033, 2020.

[17] D. K. Dheer, V. A.S., O. V. Kulkarni, and S. Doolla, "Improvement of stability margin of droop-based islanded microgrids by cascading of lead compensators," IEEE Transactions on Industry Applications, vol. 55, no. 3, pp. 3241-3251, May 2019.

[18] M. Eskandari, L. Li, M. H. Moradi, P. Siano, and F. Blaabjerg, "Active power sharing and frequency restoration in an autonomous networked microgrid," IEEE Transactions on Power Systems, vol. 34, no. 6, pp. 4706-4717, Nov 2019.

[19] A. Firdaus and S. Mishra, "Auxiliary signal-assisted droop-based secondary frequency control of inverter-based pv microgrids for improvement in power sharing and system stability," IET Renewable Power Generation, vol. 13, no. 13, pp. 2328-2337, 2019.

[20] B. Wen, D. Boroyevich, R. Burgos, P. Mattavelli, and Z. Shen, "Analysis of d-q small-signal impedance of grid-tied inverters," IEEE Transactions on Power Electronics, vol. 31, no. 1, pp. 675-687, 2016.

[21] Z. Liu, J. Liu, W. Bao, and Y. Zhao, "Infinity-norm of impedancebased stability criterion for three-phase ac distributed power systems with constant power loads," IEEE Transactions on Power Electronics, vol. 30, no. 6, pp. 3030-3043, June 2015.

[22] M. Cespedes and J. Sun, "Impedance modeling and analysis of gridconnected voltage-source converters," IEEE Transactions on Power Electronics, vol. 29, no. 3, pp. 1254-1261, 2014.

[23] B. Wen, D. Boroyevich, R. Burgos, P. Mattavelli, and Z. Shen, "Smallsignal stability analysis of three-phase ac systems in the presence of constant power loads based on measured d-q frame impedances," IEEE Transactions on Power Electronics, vol. 30, no. 10, pp. 5952-5963, Oct 2015.

[24] B. Wen, R. Burgos, D. Boroyevich, P. Mattavelli, and Z. Shen, "Ac stability analysis and dq frame impedance specifications in powerelectronics-based distributed power systems," IEEE Journal of Emerging and Selected Topics in Power Electronics, vol. 5, no. 4, pp. 1455-1465, 2017.

[25] B. Wen, D. Dong, D. Boroyevich, R. Burgos, P. Mattavelli, and Z. Shen, "Impedance-based analysis of grid-synchronization stability for threephase paralleled converters," IEEE Transactions on Power Electronics, vol. 31, no. 1, pp. 26-38, 2016.

[26] M. Amin and M. Molinas, "Small-signal stability assessment of power electronics based power systems: A discussion of impedance- and eigenvalue-based methods," IEEE Transactions on Industry Applications, vol. 53, no. 5, pp. 5014-5030, 2017.

[27] H. Liu, X. Xie, and W. Liu, "An oscillatory stability criterion based on the unified $d q$-frame impedance network model for power systems with high-penetration renewables," IEEE Transactions on Power Systems, vol. 33, no. 3, pp. 3472-3485, May 2018.

[28] L. Fan and Z. Miao, "Admittance-based stability analysis: Bode plots, nyquist diagrams or eigenvalue analysis?" IEEE Transactions on Power Systems, vol. 35, no. 4, pp. 3312-3315, 2020. 
[29] L. Harnefors, R. Finger, X. Wang, H. Bai, and F. Blaabjerg, "Vsc input-admittance modeling and analysis above the nyquist frequency for passivity-based stability assessment," IEEE Transactions on Industrial Electronics, vol. 64, no. 8, pp. 6362-6370, 2017.

[30] A. A. A. Radwan and Y. A. I. Mohamed, "Analysis and activeimpedance-based stabilization of voltage-source-rectifier loads in gridconnected and isolated microgrid applications," IEEE Transactions on Sustainable Energy, vol. 4, no. 3, pp. 563-576, July 2013.

[31] X. Wang, L. Harnefors, and F. Blaabjerg, "Unified impedance model of grid-connected voltage-source converters," IEEE Transactions on Power Electronics, vol. 33, no. 2, pp. 1775-1787, 2018.

[32] D. Lu, X. Wang, and F. Blaabjerg, "Impedance-based analysis of dc-link voltage dynamics in voltage-source converters," IEEE Transactions on Power Electronics, vol. 34, no. 4, pp. 3973-3985, 2019.

[33] F. Cavazzana, A. Khodamoradi, H. Abedini, and P. Mattavelli, "Analysis of an impedance modeling approach for droop-controlled inverters in system dq frame," in 2019 IEEE Energy Conversion Congress and Exposition (ECCE), Sep. 2019, pp. 5576-5583.

[34] S. Wang, Z. Liu, J. Liu, D. Boroyevich, and R. Burgos, "Smallsignal modeling and stability prediction of parallel droop-controlled inverters based on terminal characteristics of individual inverters," IEEE Transactions on Power Electronics, vol. 35, no. 1, pp. 1045-1063, Jan 2020.

[35] M. Mao, Y. Ding, Y. Shen, and L. Chang, "Hybrid impedance-based modelling and stability analysis of img-picdps," in 2017 IEEE Energy Conversion Congress and Exposition (ECCE), Oct 2017, pp. 3353-3358.

[36] M. Dokus and A. Mertens, "Sequence impedance-based stability analysis of droop-controlled ac microgrids," in 2019 IEEE 10th International Symposium on Power Electronics for Distributed Generation Systems (PEDG), 2019, pp. 768-773.

[37] C. Zhang, M. Molinas, A. Rygg, and X. Cai, "Impedance-based analysis of interconnected power electronics systems: Impedance network modeling and comparative studies of stability criteria," IEEE Journal of Emerging and Selected Topics in Power Electronics, pp. 1-1, 2019.

[38] A. Rygg and M. Molinas, "Apparent impedance analysis: A small-signal method for stability analysis of power electronic-based systems," IEEE Journal of Emerging and Selected Topics in Power Electronics, vol. 5, no. 4, pp. 1474-1486, 2017.

[39] B. Gustavsen and A. Semlyen, "Rational approximation of frequency domain responses by vector fitting," IEEE Transactions on Power Delivery, vol. 14, no. 3, pp. 1052-1061, 1999. 